Arteterapia. Papeles de arteterapia y educación para inclusión social ISSN-e 1988-8309

\title{
Cuerpo emocional y trauma: guía terapéutica expresivo-corporal en la construcción del autoconcepto e imagen proyectada en mujeres que han sufrido maltrato
}

\author{
Rosaura Navajas Seco ${ }^{1}$
}

Recibido: 21 de Julio de 2017/ Aceptado: 16 de agosto de 2017

Resumen. En este artículo se presenta la gran labor que desempeña el arte y la expresión corporal en la recuperación y la mejora del autoconcepto e imagen corporal en la mujer con trauma que sufre maltrato. Se plantea una forma de trabajo que dará la clave para realizar la aplicación de programas terapéuticos basados en contenidos expresivo corporales con el objetivo de construir una imagen corporal renovada y respetada. Todo ello se realiza con propuestas creativas en donde el cuerpo y movimiento son los ejes principales del trabajo en la mejora y conocimiento de uno mismo y su relación con los demás.

Palabras clave: trauma-mujer; terapia-expresivo corporal; mujer-imagen corporal

[en] Emotional body and trauma: body language therapeutic guide in the construction of self-concept and image projected in women who have suffered abuse

Abstract. This article presents the great work that art and body/ corporal expression play in the resilien-
ce and improvement of the self-concept and body image in women suffering trauma abuse. It introduces
a way of working which will be the key for the implementation of therapeutic programmes based on
body expressive contents with the aim of building a renewed and respected body image. This will take
place on the basis of creative proposals where the body and the movement are the main axes for wor-
king in the improvement and the knowledge of oneself and her relationship with others. Key words: Trauma-woman; therapy-body expression; woman-body image.

Sumario. 1. Contribución de la Expresión Corporal como terapia para la mejora del autoconcepto en mujeres con trauma por maltrato. 2. Definiciones sobre Expresión Corporal en relación con el término Autoconcepto. 3. Similitudes conceptuales (Expresión Corporal-Autoconcepto). 4. Referencias bibliográficas.

Cómo citar: Navajas Seco, R. (2017). Cuerpo emocional y trauma: guia terapeutica expresivo-corporal en la construcción del autoconcepto e imagen proyectada en mujeres que han sufrido maltrato, en Arteterapia. Papeles de arteterapia y educación para inclusión social 12, 219-231. 


\section{Contribución de la Expresión Corporal como terapia para la mejora del autoconcepto en mujeres con trauma por maltrato}

Relacionando el término Expresión Corporal con la palabra autoconcepto, se puede observar cómo ambos de forma muy especial contribuyen a la mejora del ser humano en todo tipo de situaciones cuando se trabajan conjuntamente. Cada vez se ve más necesario relacionar estos dos conceptos con la psicología y ver las influencias que tienen, porque la Expresión Corporal en muchos casos está actuando como terapia y aparecen técnicas corporales que están ayudando al equilibrio psíquico-emocional, Artilla (2013). Numerosos autores desde la Expresión Corporal están poniendo de manifiesto la importancia de esta temática dentro de la dimensión emocional que contribuye al enriquecimiento del individuo (Arriagada, 2004).

Tanto es así que se incluirá en muchos programas como medio de herramienta, contenido u otras opciones para ayudar a personas que tienen problemas de tipo emocional a través de actividades que están relacionadas con la danza terapia, Abellán y Martínez-Illescas (2002). Este tipo de actividades son importantes porque favorecen el desarrollo integral del individuo a través de potenciar aspectos creativos y de sensibilidad para incrementar la capacidad expresiva, Dowmat, (2002). Las investigaciones incluyen temáticas sobre el análisis del cuerpo y las posibilidades, identificando gestos, acciones y actitudes que caracterice a los individuos y así poder conocerse más, Álvarez (2003).

La Expresión Corporal va teniendo más cuerpo dentro de la línea de actuación pedagógica donde autores como Villard (2009), constatan la importancia de darle consistencia a este contenido por su peso específico en la mejora del desarrollo integral del individuo. Ruano (2004), en una investigación que hace con distintos grupos de universitarios, concluye que tras realizar la asignatura los grupos a los que ella dio clases disminuyó la vergüenza y se mejoró el nivel de habilidad social tanto en hombres como en mujeres. Trabajos realizados para la mejora del autoconcepto utilizan el arte como medio expresivo para liberar y expresar emociones con el objetivo también de unir muchas temáticas que pueden ir en torno al desarrollo del autoconcepto y su mejora (Cutillas, 2005).

El teatro como uso liberador estratégico en las clases y como ejemplo el teatro negro surgido en China, que se ha transferido a espectáculos de magia (Pedraza y Ruiz, 2006). El teatro favorece la confianza y la autoestima en la persona, porque cuando se realiza proporciona satisfacciones sobre todo de tipo emocional e intelectual ya que se trabaja en colaboración y cooperación de forma activa y participativa (Dougill 1987; Lazar 1993; Stern 1980; Torres, 1996; Wessels 1987). La Expresión Corporal trabaja de forma integral y da gran importancia al contacto entre personas, donde se generan situaciones de afectividad, del intelecto, del conocimiento y del trabajo en grupo en donde se promueven actitudes y valores para la vida (Tamayo y Vázquez, 2004). La Expresión Corporal siempre estará ligada a la emocionalidad por su carácter y por las situaciones que se crean, Levas (2014). 


\section{Definiciones sobre Expresión Corporal en relación con el términoAutoconcepto}

Como dicen Calvo, Ferreira, León García (2011), la Expresión Corporal es complicada en su definición y hay una necesidad para ponerse de acuerdo en concretar su concepto. Depende desde dónde esté ubicada esa definición así se le otorga diferentes tipos de significados. Además se encuentra entre uno de los contenidos más desplazados y que menos quieren dar los profesionales en el sistema educativo (López y Gutiérrez, 2002), por lo que aun su grado de influencia no es grande para los que no la conocen, "nadie que no entienda o controle la Expresión Corporal debe opinar sobre ella, ya que puede desvirtuar el contexto y por supuesto, las conclusiones" (Calvo, Prados y Sánchez, 2011, p.22), es desde la infancia y adolescencia donde estos conceptos deben ser aprendidos para una construcción de autoconcepto e imagen corporal ajustados.

La Expresión Corporal, se presenta como el medio más antiguo que han tenido los seres humanos para comunicarse, anterior al lenguaje escrito y hablado, donde se han podido expresar tanto sensaciones, sentimientos, como emociones y pensamientos para entrar en contacto con el medio y con los demás individuos, (Verde, 2005). La definición del término queda muy abierta dependiendo de muchos aspectos y del campo donde venga, del tipo de práctica que se haga con ella, o del objetivo o función que se le dé según el fin. El lenguaje corporal aparece en nuestras vidas desde el momento que venimos al mundo, el lenguaje corporal pasa por muchas fases, desde los inicios se hará necesario para comunicarse con el medio y por la ausencia de un lenguaje verbal codificado. Es entonces cuando todo tipo de expresiones nacidas de forma consciente e inconsciente pone al sujeto en contacto con su entorno y es con este desde el que se empieza a entender que la manifestación expresiva de cualquier gesto hace relacionar a la persona con otros. Así es como Lapierre (1985), habla de la importancia de la Expresión Corporal como medio fundamental de comunicación con las personas.

Independientemente del contexto, objetivos y otros aspectos donde se suele ubicar la Expresión Corporal, también se reúne una gran cantidad de definiciones venidas de diferentes autores que tienen que ver con las experiencias o vivencias de las personas que definen el término de Expresión Corporal. Así Bara (1975), considera a la Expresión Corporal como un contenido que revela los estados internos de la persona, sin que importe la estética o que tenga como objetivo consciente el expresarse como única función. Para Motos (1983), sin embargo sí que es importante reconocer que la Expresión Corporal viene definida por un conjunto de técnicas que utiliza el ser humano como elemento de comunicación y que este tipo de lenguaje revela un contenido de naturaleza psíquica.

Berge (1985) también habla de la Expresión Corporal como un camino para encontrar y adivinar, aspectos profundos y de carácter auténtico sobre nosotros mismos. Según Bossu y Chalaguier (1986), encuentra la definición de Expresión Corporal como una forma original de expresión que tiene sus propias justificaciones y métodos de trabajo en su representación. Riveiro y Schinca (1995), lo conceptúa como una materia en donde el cuerpo de forma intencional y a través del estudio sobre gestos, actitudes y formas se pone en contacto con el mundo externo. Romero (1999) encuentra en la Expresión Corporal, una finalidad de tipo expresivo, comunicativo y estético, que tiene que ver con el cuerpo, el movimiento y el sentimiento como instrumentos base del desarrollo motor. También la Expresión Corporal ha 
sido considerada como un arte donde se utilizan técnicas a través del cuerpo y del movimiento que sirven para liberar energía, según Ortiz (2002).

En esta línea Stokoe, nos habla también de la Expresión Corporal como un conjunto de técnicas que utiliza el cuerpo y el movimiento para mejorar en el ser humano su desarrollo en la formación integral y como decía Ortiz (2002), esta disciplina sirve como liberación en el ser humano. Harf y Stokoe (citado por Ortiz, 2002). Para Motos (1983), la técnica es uno de los pilares más importantes de la Expresión Corporal. Considera que no se puede prescindir de ella, aunque otros autores piensen que es más importante llevar una metodología adecuada que no limite y que ayude a expresar mejor el yo interno, sus actitudes, sentimientos y emociones (Pérez, 2005). Hay otros autores/as que sitúan a la Expresión Corporal como una disciplina, es el caso también de Romero (1997) y Schinca (1988), cuyo objetivo dentro de la conducta motriz es que tenga una finalidad expresiva, comunicativa y estética que incluye una visión corporal global donde se entremezcla tanto lo cognitivo, afectivo como psicomotor.

El cuerpo casi siempre se encuentra presente en las definiciones del concepto Expresión Corporal, así Villada (1997) habla de éste como el transmisor de sentimientos, pensamientos, emociones, deseos que se producen de forma natural y espontánea y se exteriorizan hacia fuera. La Expresión Corporal manifiesta a través del cuerpo el estilo peculiar de cada persona expresando su relación con otros y con el mundo (Santiago, 1985). Podemos observar la unión tan estrecha que existe entre la Expresión Corporal y el autoconcepto, en ambas se repite contantemente la presencia del cuerpo y la expresión de emociones, sentimientos, sensaciones y actitudes. El cuerpo expresivo y comunicativo trata de establecer la relación con el mundo que le rodea a través de todas las percepciones que siente de lo que le dice su entorno (Clemes y Bean, 1996; Clark, Clemes y Bean, 2000). Ese cuerpo también es creativo y de ahí otro punto en común entre Expresión Corporal y autoconcepto, la necesidad de la creación que forma parte de la idea de autores tales como Sánchez, Coterón, Padilla, Llopis y Montávez (2008). Sierra y Cachadiña (2008), ven como crucial que uno de los objetivos de Expresión Corporal sea que el ser humano consiga un nivel de creatividad importante, y para ello se debe alentar a desarrollar propuestas que generen este tipo de aprendizajes que mejoren la construcción de la imagen corporal.

La Expresión Corporal ha sido uno de los ámbitos que en mayor medida se han interesado por la creatividad, Canales y López (2002) consideran que la creatividad está vinculada a la Expresión Corporal, como algo necesario e inevitable en este campo, porque continuamente se tiende a estimular la singularidad de cada individuo a través de los procesos de desarrollo con actividades en las que se incluyen ese tipo de trabajos que necesitan de esos procesos creativos.

\section{Similitudes conceptuales (Expresión Corporal-Autoconcepto)}

Este trabajo pone de manifiesto las grandes similitudes que tiene el autoconcepto en relación con la Expresión Corporal. Empezaremos porque en las definiciones de sus términos hay puntos en común muy parecidos ya que en ambos existe una gran conexión entre la construcción social y las relaciones interpersonales (Harter, 1999). También se habla de lo importante que es para el sujeto, el tipo de informaciones 
que recibe del entorno (Graig y Baucum, 1992) y de las maneras en las que estos mensajes son acogidos (Gallagher, 1999). La Expresión Corporal es un campo ideal para el trabajo del autoconcepto porque se pone a prueba en las personas cual es el nivel del estado de su autoconcepto, el sujeto al ser social recibe informaciones que le hacen conocer más de su persona (Martorel, 1988).

El carácter multidimensional de los dos constructos es obvio (Harter, 1985). La psicología, la antropología, la biología y muchas ciencias más están al lado del autoconcepto, lo mismo le pasa a la Expresión Corporal, que además de incluir todas las citadas, está el arte, la danza, el teatro, la gimnasia, etc. Ambos necesitan de muchos campos para ser comprendidos, por ello se pueden aplicar en cualquier ámbito y sobre todo en bastantes áreas (Pérez y Urdampilleta, 2012). En ambos el cuerpo se toma como una unidad funcional que reúne aspectos físicos, intelectuales, sociales y espirituales (Ruano, 2004). El cuerpo no es divisible ni para el autoconcepto, ni para la Expresión Corporal, además es un cuerpo que se entiende como libre, crítico y creativo enfocado a la autonomía (Learreta, Ruano y Sierra, 2005). Tanto uno como el otro se nutre de las emociones, sentimientos y sensaciones, (Arteaga, Viciana y Conde, 1997; González, 1982). Igual que la Expresión Corporal ha sido siempre un añadido esporádico de corte marginal en diferentes ámbitos (García, Bores y Martínez, 2007) el autoconcepto no ha sido tema de trabajo incluido como crucial, sobre todo en edades tempranas, sino que se presta a ser tratado solo cuando existe un foco concreto de trabajo. Para que estos dos conceptos se puedan desarrollar con armonía es necesario que se creen climas confortables donde se presten a la espontaneidad, flexibilidad, imaginación, originalidad de las situaciones (Llopis, 2010; Trigo, 2001; Villada, 1997) para que se consigan mejoras sobre el trabajo del autoconcepto y en la Expresión Corporal. Por lo tanto la multidimensionalidad, el considerarlos como unidades dotadas de significado, la necesidad de que tengan que ver con muchas ciencias y campos para definirse y que se desarrollen en climas motivacionales adecuados para su funcionamiento y mejora dejan entrever lo importantes y necesarios que son estos dos constructos en la formación del individuo, según se ha visto en la literatura.

\section{APLICACIÓN TERAPÉUTICA DESDE EL CAMPO EXPRESIVO-CORPORAL}

Nuestro modelo de trabajo se fue elaborando tras los años con la toma de conciencia de aquellos focos donde había que poner especial atención con mujeres que sufrieron maltrato y cargaban con traumas aún sin resolver. Tras la observación de sus comportamientos, de sus actitudes, de sus verbalizaciones y sobre todo de la imagen corporal proyectada, había datos que se repetían y sobresalían de nuestros escritos como relevantes para preparar acciones en la mejora del autoconcepto y construcción de una imagen corporal ajustada como objetivos principales.

Se pudieron analizar diferentes componentes como categorías reinas importantes para estudiarlos desde distintas perspectivas, llegando a hacer un diagnóstico bastante elaborado sobre la situación de la mujer que sufre maltrato unido al trauma que presenta. Los componentes más destacados fueron:

* Componente cognitivo

* Componente afectivo

* Componente conativo 
A continuación se presenta una matriz de análisis previa a la realización de cualquier programación en donde tenemos como propósito mejorar el estado de la mujer con trauma y sus condiciones de vida. Es la fase previa de la programación para conocer el estado de la cuestión además de su fundamentación para proceder a desarrollar una terapia ajustada a las características de cada persona. Si conocemos su estado cognitivo, su estado emocional y su forma de proceder en actos, habremos conseguido identificar emociones, comportamientos y actitudes para posible modificación o mejora de ellas y su actitud, en definitiva ir eliminando distorsiones y mejorando su estado general.

\begin{tabular}{|c|c|c|}
\hline \multicolumn{2}{|c|}{ MATRIZ DE ANÁLISIS } \\
"YO" PERCEPCIÓN DE UNO MISMO \\
(ANÁLISIS DE DISTORSIONES DE CADA COMPONENTE) \\
\hline ¿Cómo soy? & ¿Cómo siento? & ¿Cómo actúo? \\
\hline PIENSO & SIENTO & ACTUO \\
\hline COMPONENTE & COMPONENTE & COMPONENTE \\
CONATIVO
\end{tabular}

Tabla 1. Elaboración propia

\section{MATRIZ DEL “YO” PERCEPCIÓN DE UNO MISMO}

El componente cognitivo.- Nos es representado por la pregunta ¿cómo soy?, y lo llamamos "Cuerpo pensante". Este cuerpo pensante está relacionado con el autoconcepto de la persona, la percepción que se tiene de uno mismo y qué partes de esa percepción se suponen dañadas, con pensamientos distorsionados o no ajustados a la realidad. Este autoconcepto tiene dos partes muy visibles de interés terapéutico en el campo expresivo-corporal y son la parte física, determinada por la imagen corporal (morfología corporal, rasgos faciales, alimentación, higiene. etc) y aspecto personal (actitud postural, gestos, movimientos, vestuario y ornamentación corporal entre otras). Dentro del autoconcepto, el autoconocimiento es parte importante sobre todo porque trata la aceptación de uno mismo, las influencias y la aceptación de éstas como las reformulaciones que realizamos personales y las autoafirmaciones. Todos estos términos están relacionados con el componente cognitivo por lo tanto son importantes a tener en cuenta en cualquier aplicación de programa terapéutico. 
El componente afectivo.- Nos es representado por la pregunta ¿cómo siento?, y lo llamamos "cuerpo sensitivo". Este cuerpo sensitivo está relacionado con la autoestima, donde la persona entra en examinar su parte más relacionada con (los actos, las capacidades, los atributos), las percepciones vienen por parte del sentimiento de competencia sobre nuestras acciones. En la mujer con trauma esta percepción se ve anulada y en muchos casos por la sensación de impotencia de no saber evaluar de forma ajustadas cuáles son sus verdaderas competencias y limitaciones. En este cuerpo sensitivo se comparan criterios y valores personales a partir de las experiencias, el entorno y otros significados. Las temáticas a tratar o los conceptos dentro de este componente, está la aceptación de los logros, la autonomía, asumir riesgos y responsabilidades, la aceptación de frustraciones, trabajar en la creatividad y curiosidad por las cosas, afrontar retos, poder tener capacidad de influenciar en otros, etc.

El componente conativo.- Nos es representado por la pregunta ¿cómo actúo?, y lo llamamos "cuerpo comportamental". Este cuerpo comportamental está relacionado con la autoeficacia, por lo tanto es un cuerpo que actúa, que se mueve, que se comunica a través de acciones conscientes pero que en la mayoría de los casos es un cuerpo que expresa el trauma de distintas maneras observables para el evaluador de imagen corporal y su proyección. Dentro de este componente es importante hacer un análisis de los modos de comportamiento y actuaciones en la mujer con trauma, y de la manera en que ve como sus acciones se ven comprometidas sobre todo por la aceptación de otros. La pérdida de capacidad de presentarse con autoafirmación sobre sus actos. Es un cuerpo que se rebela a través del movimiento, y las temáticas que se trabajan son el tipo de conductas, las manifestaciones corporales, la proyección de la imagen corporal en cada comportamiento, etc.

\section{“YO” PECEPCIÓN DE UNO MISMO CUERPO EXPRESIVO}

Una vez analizada la matriz y considerando cada componente y cómo se articulan entre ellos y las relaciones que guardan, ya sabemos en el marco que nos movemos y el estado de la cuestión de cada caso. Por lo tanto así podremos actuar con más precisión en la aplicación de nuestro programa terapéutico expresivo-corporal. Se elige utilizar como contexto de interés la expresión corporal, porque entre otras cosas sus ejes principales de actuación son el cuerpo y el movimiento, de crucial importancia para la construcción de una buena imagen corporal en la mujer con trauma. En el cuerpo se dan todos los componentes analizados y a través del movimiento conocemos la manera de actuar que tiene ese cuerpo según experiencias vitales en relación con las peculiaridades que tiene la persona, como la manera de conocerse, de emocionarse y de actuar en consecuencia. La expresión corporal nos permite en este campo no solo saber la importancia que cada individuo le da a su cuerpo, sino que también es observable como ese cuerpo maneja el movimiento a través de sus acciones y actitudes corporales donde existen señales no solo físicas sino simbólicas de sus experiencias de vida. 


\section{TERAPIA: VIVENCIAR A TRAVÉS DEL CUERPO EMOCIONES Y SENTIMIENTOS GUIA DE TRABAJO}

Componente cognitivo.- El trabajo de análisis de nuestro cuerpo brinda la oportunidad de conocer nuestro ser más interno.

\section{Características corporales y aspecto personal}

- A través de la observación de nuestra morfología corporal entendemos que nuestro cuerpo es diferente a otros por ser único e irrepetible. Sus formas nos dan información sobre muchos aspectos tales como etnia, sexo, alimentación, tipo de belleza, higiene, prácticas deportivas, etc, que el entorno recibe y así interactúa con él.

- Un análisis crítico constructivo de la realidad que cada individuo observa de sí mismo, hace entender el porqué de muchas de las interacciones que se establecen con su entorno.

- El investigar sus posibilidades y limitaciones entraña que tengamos una visión más clara de lo que nos sucede en relación con el mundo externo.

- Nuestro cuerpo ocupa un espacio y unas trayectorias cuando está en movimiento. Por lo tanto no somos invisibles a los demás y debemos analizar nuestra representación en el espacio respecto a los otros. Los movimientos caracterizan una forma de ser y vivir que lo diferencian de otros.

- La imagen corporal que proyectamos es evaluada por los demás recibiendo críticas según nos ven. Esa concepción estereotipada de fuera la recibimos de una forma u otra dependiendo de cómo vivenciemos esa experiencia.

$\underline{\text { Será interesante trabajar en este apartado: }}$

- La influencia de los cánones de belleza

- La importancia que le damos nosotros al tipo de imagen que la sociedad entiende como perfecta.

- Se tendrá que realizar ajustes entre la imagen y características que tenemos y las que quisiéramos tener.

- Ver si existen distorsiones en la percepción del yo corporal real.

- Ayudar a que nuestro aspecto personal mejore con nuestra observación y cuidado constante. Ser conscientes de nuestro cuerpo.

- Atender a aspectos informativos sobre la alimentación y la importancia de ésta sobre nuestro cuerpo. La dieta será un factor determinante de la salud y de nuestro bienestar.

\section{Aplicado al campo expresivo-corporal:}

- Sobre la observación directa de nuestro cuerpo a través de un espejo o con otros entenderemos cómo somos. Jugar con nuestra cara y cuerpo deformándolo, tocándolo, viéndolo conscientemente nos abrirá puertas para conocernos internamente. 
- Sensoriales percibiremos con otras nuestros formas corporales, el sentido del tacto, del olor nos información de la otra persona.

- Analizamos nuestros rasgos característicos y cómo los describimos y como nos vemos en esa descripción.

- Expresaremos nuestros deseos de cambio de aquellas cosas de nuestro cuerpo que son modificables y veremos en aquellas donde existe limitación.

- Aceptaremos aquello que es imposible cambiar, pero desviaremos nuestra atención para darle sentido a otras cualidades y características que son bonitas en nosotros.

\section{Objetivo principal: PENSAR SOBRE EL CUERPO}

Interpretar nuestro cuerpo, identificándolo, observando su imagen, sus movimientos, siendo conscientes de la imagen que proyectamos y que queremos proyectar.

Componente emotivo.- Sentir el cuerpo hace despertar nuestras emociones. La necesidad de atender nuestro interior posibilita que la persona viva más y mejor todas las experiencias que tengamos.

\section{Examinar nuestros actos, capacidades y atributos:}

- La necesidad de expresar nuestras emociones es un primer paso para reconocer que somos capaces de aprender a través de la verbalización de nuestros sentimientos. El ser conscientes que vivimos en un cuerpo que siente posibilita que seamos capaces de saber más de nosotros.

- El cuerpo como trasmisor de sentimientos y herramienta de expresión exterior de lo que nos ocurre en el interior hace que seamos más conscientes de cómo somos.

- El análisis de nuestro cuerpo y de lo que transmite con su actitud corporal, movimientos y gestos puede acercarnos a conocer más de nosotros. Los sentimientos se transcriben corporalmente en formas inconscientes de estar y presentarnos a los demás.

- La importancia de investigar sobre lo que nuestro cuerpo nos dice y habla a través de la interacción con el medio puede fundamentar un conocimiento más profundo de nuestra personalidad y formas de ser.

- El cuerpo nos explica cómo somos, solo hace falta atender a sus necesidades y escucharle. Es un trabajo de observación no solo de nosotros mismos sino de los demás para comparar y aprender del otro.

- La expresión corporal de nuestro cuerpo hace constatar nuestros atributos como personas que sentimos y vivimos una forma peculiar, original e individual de existir.

- Expresar nuestros sentimientos posibilita que seamos capaces de analizarlos y modificar aquellos que nos hacen daño. La forma constatable de que existen es que se expresan a través de múltiples acciones tales como movimientos, gestos y actitudes que nos delatan.

- El conocer nuestros sentimientos y analizarlos posibilita que los podamos controlar y modificar. El cuerpo es una herramienta de trabajo que puede hacer que esto suceda. 
$\underline{\text { Será interesante trabajar en este apartado: }}$

- La expresión de sentimientos tanto verbal como corporalmente.

- La fluidez de sentimientos sin que existan criticas ni juicios sobre ellos.

- La importancia del cuerpo como herramienta de trabajo en la expresión de sentimiento.

- El análisis de sentimientos que nos perjudican para modificarlos.

\section{Aplicado al campo expresivo-corporal:}

- Utilizaremos el cuerpo en expresar conscientemente diferentes estados de ánimo, actitudes, formas de ser.

- Evaluaremos nuestro cuerpo, lo que dice y nos dice.

- Compararemos diferentes actitudes y lo que pueden expresar.

- Relacionaremos movimientos con distintos estados de ánimo y el significado de esos movimientos.

\section{Objetivo principal: SENTIR EL CUERPO}

Componente conativo.- El cuerpo da respuesta a nuestras emociones y nuestros pensamientos, solo hay que dejarle que fluya. Nuestro lenguaje corporal es fiel a nosotros mismos y a nuestra condición como ser humano.

\section{Examinar nuestros comportamientos y actuaciones:}

- El cuerpo es el encargado de expresar todo o casi todo lo que ocurre en nuestro interior y exterior. Abarca aspectos biopsicofísicos. Como nos encontramos de salud, cual es nuestra morfología y forma de presentarnos, cuál es su estética y cuál es su lenguaje corporal.

- En el análisis y tratamiento del cuerpo cuando actúa es donde encontramos la posibilidad de cambios de comportamiento beneficiosos para modificar conductas.

- El análisis profundo de nuestros movimientos, gestos y actitudes hace que podamos ser capaces de modificar aspectos relacionados con la forma de pensar y de sentir.

- Constatar que la modificación y el control corporal puede dar información al exterior de lo que está pasando en nuestro interior es un hecho fundamental para producir cambios sobre otros/as. Solo nos conocemos y nos conocen a través de lo que el cuerpo dice.

\section{Será interesante trabajar en este apartado:}

- La importancia de nuestro cuerpo como herramienta de trabajo para la modificación de conductas.

- La importancia de nuestro cuerpo como transmisor de sentimientos.

- El cuerpo para demostrar que corporalmente hay gestos, movimientos y actitudes que manifiestan el estado emocional en el que nos encontramos. 
- Entender la contradicción de lo que en muchos momentos comunica el cuerpo y lo que pensamos.

- Saber que un cuerpo equilibrado de pensamiento y emociones es capaz de ser plástico, estético y creativo.

- El cuerpo como barrera y limitación en cuanto a la expresión de lo que dice nuestro interior.

- Constatar que el cuerpo es liberador de emociones y estado de ánimo a la vez que puede ejercer de bloqueante para los que están a tu alrededor.

\section{Aplicado al campo expresivo-corporal:}

- Tomaremos conciencia de nuestro cuerpo a través de nuestras formas de expresión,

- Veremos las posibilidades que nos ofrece nuestro cuerpo expresivo para modificar conductas que no nos beneficien en la relación con otros/as.

- Utilizaremos técnicas de control corporal para ajustar posibles distorsiones entre pensamiento y acto.

- Trabajaremos actividades de respiración para que el cuerpo se oxigene y sea capar de liberar tensiones y bloqueos.

- Investigaremos sobre otros cuerpos para ver conductas semejantes y diferentes a las nuestras.

\section{Objetivo principal: ACTUAR CON EL CUERPO}

\section{TRABAJO INTERDISCIPLINAR: FUSIÓN DE DISCIPLINAS EL ARTE DE LA PINTURA Y EL MOVIMIENTO}

Es interesante combinar diferentes disciplinas para enriquecer la aplicación de programas de ayuda a la mujer. Se observa que fusionar actividades de pintura con la utilización del cuerpo y movimiento para transferir emocionalmente estados internos es una herramienta poderosa que mejora y estabiliza los estados tanto físicos como psíquicos de la persona. Posibilita la liberación de estados emocionales sin ser conscientes pero que sanan y tranquilizan tanto la mente como el cuerpo. Se genera una catarsis y aún más cuando se hace en unión con otras personas donde se da tanto afinidad como empatía en los procesos de interacción.

La fusión de diferentes códigos comunicativos completan el mapa de socialización entre las personas que se encuentran haciendo programas de estas características. Poder canalizar los sentimientos de culpa, impotencia, resignación, desconfian$\mathrm{za}$, rabia y otros muchos, junto con la expresión de sensaciones de futuro desolador, anulación de emociones, pérdida de poder, estado de angustia, de alarma, dispersión, frustración y mucho más es absolutamente terapéutico y mejora el equilibrio físico-psíquico y emocional-relacional de la mujer con trauma por maltrato.

Esta mejora se ve acrecentada cuando se fusiona el arte y el movimiento para reflejar los estados internos emocionales que afloran cuando interpretan sus propias realidades, causando verdadera conciliación entre su yo más interno y la imagen corporal proyectada hacia los demás.

Se trabajan diferentes códigos comunicativos utilizando todos los sentidos para incorporar nuevos sentimientos en positivo que haga que desde el arte y el trabajo 
expresivo corporal modifiquen las emociones y a su vez interpreten la realidad desde otras perspectivas abriendo su mente y creando un mundo de sensaciones diferente.

Cuanto más interacción entre disciplinas, más enriquecimiento, en el saber, saber ser y saber hacer, que son los saberes por donde se debería afrontar de forma transversal cualquier tipo de terapia que de una formación integral y donde tenga sentido conjunto. La capacidad de tener habilidades y expresarlas, la capacidad de poder emocionarse con libertad y la capacidad de que todos los conocimientos sean prácticos y reales en la vida para sentir que cada momento tiene significado y merece la pena vivirlo.

En conclusión.- El objetivo en definitiva es que desde diferentes disciplinas, con un conocimiento previo de análisis de la realidad, considerando la transversalidad de saberes y teniendo una visión integral de afrontamiento del trauma, la persona sea capaz de vivir con calidad y sienta la vida como un privilegio. Siendo capaz de afrontar diferentes realidades y superando obstáculos con estrategias creativas que le hagan mejorar como persona.

\section{Referencias bibliográficas}

Álvarez, M. J. (2003). La comprensión de los gestos corporales básicos en el ámbito de la expresión corporal. Tándem: Didáctica de la Educación Física, 4 (13), 79-91.

Archilla, M. T. (2013). Dificultades del profesorado de Educación Física con los contenidos de Expresión Corporal en Secundaria (tesis doctoral) Univesidad de Valladolid, Valladolid.

Arriagada, M. T. R. (2004). La influencia de la expresión corporal sobre las emociones: un estudio experimental (tesis doctoral). Universidad Politécnica de Madrid, Madrid.

Bara, A. (1975). La expresión por el cuerpo. Buenos Aires: Búqueda.

Berge, I. (1985). Vivir tu cuerpo. Para una pedagogía del movimiento. Madrid: Narcea.

Bossu, H., y Chalaguier, C. (1986). La expresión corporal, método y práctica, Barcelona: Martínez Roca.

Calvo, Á., Ferreira, M., León, J. A., y García, I. (2011). Un análisis DAFO sobre expresión corporal desde la perspectiva de la educación física actual. EmásF: Revista Digital de Educación Física, 11, 20-28.

Canales, I., y López Villar, C. (2002). Creatividad y singularidad, ejes vertebradores de la expresión corporal. Revista de Educación Física, 86, 23-26.

Clark, A., Clemes, H., y Bean, R. (2000). Cómo desarrollar la autoestima en adolescentes. Madrid: Editorial Debate.

Clemes, H., y Bean, R. (1996). Cómo desarrollar la autoestima en los niños. Madrid: Editorial Debate.

Coterón, J., y Sánchez, G. (2012). Expresión corporal en Educación Física: Construcción de una disciplina. EmásF: revista digital de educación física, 14, 164-175.

Cutillas, V. (2005). La enseñanza de la dramatización y el teatro: Propuesta didáctica para la enseñanza secundaria (tesis doctoral). Universitat de València, Valencia.

Dougill, J. (1987). Drama Activities for Language Learning. London: Macmillan.

Dowmat, L. P. (2002). Yo puedo, tú puedes... La Expresión Artística como puente de comunicación para personas con discapacidad. Arte, Individuo y Sociedad, Anejo I, 405-409. 
Lapierre, A. (1985). El adulto frente al niño de 0 a 3 años. Barcelona: Editorial Científico-Médica.

Lazar, G. (1993). Literature and Language Testing: A guide for teachers and trainers. Cambridge: Cambridge University Press.

Levas, R. (2014). Fundamentación teórica y propuesta de intervención sobre Expresión Corporal orientada al trabajo emocional (trabajo fin de grado). Universidad de Valladolid, Valladolid.

Martínez, A. C., Chillón, P., Martín-Matillas, M., Pérez López, I., Castillo, R., Zapatera, B., Vicente-Rodríguez, G., Casajús, J. A., Álvarez-Granada, L., Romero Cerezo, C., Tercedor, P., Delgado-Fernández, M. (2012). Motivos de práctica de actividad físico-deportiva en adolescentes españoles: Estudio AVENA. Profesorado. Revista de currículum y formación de profesorado, 16 (1), 391-398.

Motos, T. (1983). Iniciación a la expresión corporal. Barcelona: Humanitas.

Ortiz, M. M. (2002). Expresión Corporal. Una propuesta didáctica para el profesorado de Educación Física. Granada: Grupo Editorial Universitario.

Pérez, D. M. (2005). La expresión corporal en el currículum: Una propuesta integradora desde la formación del profesorado hasta el primer ciclo de la enseñanza primaria (tesis doctoral). Universidad de Granada, Granada.

Riveiro, L. y Schinca, M. (1995). Optativas: Expresión Corporal. Madrid: Ministerio de Educación y Ciencia.

Romero, R. (1999). La expresión y comunicación corporal desde el ámbito de la educación física: Elementos, características y enfoque globalizador de la misma. Granada: Asociación para el desarrollo de la Comunidad Educativa en España y Grupo Editorial Universitario.

Ruano, M. T. (2004). La influencia de la expresión corporal sobre las emociones: Un estudio experimental (tesis doctoral). Universidad Politécnica de Madrid, Madrid.

Santiago, P. (1985). De la expresión corporal a la comunicación interpersonal. Madrid: Narcea.

Sierra, M. A., y Cachadiña, M. P. (2008). Un cuerpo creativo mejora tu expresión. Actas del II Congreso Internacional de Expresión Corporal y Educación. Salamanca: Amarú.

Stern, S. L. (1980). Drama in second language learning from a psycholinguistic perspective. Language Learning, 30 (1), 77-97.

Tamayo, J. A., y Vázquez, R. A. (2004). Utilización de la expresión corporal como medio de enseñanza de los diferentes contenidos del área de educación física en la escuela. Lecturas: Educación física y deportes, 77, 22.

Torres, J. J. (1996). Nuevos horizontes para el teatro en la enseñanza de idiomas. Almería: Servicio de Publicaciones de la Universidad de Almería.

Verde, F. (2005). Expresión corporal, movimiento, creatividad, comunicación y juego. Madrid. Editorial Síntesis.

Villada, P. (1997). Expresión corporal. Madrid: Pila Teleña.

Villard, M. (2009). La expresión corporal, un camino tortuoso. EmásF, Revista Digital de Educación Física, 14, 9-26.

Wessels, A. C. (1987). Drama. Oxford: Oxford University Press. 